\title{
Long Term Verification of Glucose-Insulin Regulatory System Model Dynamics
}

\author{
J. Lin ${ }^{1}$, J. G. Chase ${ }^{1}$, G. M. Shaw ${ }^{2}$, T. F. Lotz ${ }^{1}$, C. E. Hann ${ }^{1}$, C. V. Doran ${ }^{1}$, D. S. Lee ${ }^{3}$ \\ ${ }^{1}$ Department of Mechanical Engineering, University of Canterbury, Christchurch, New Zealand \\ ${ }^{2}$ Department of Intensive Care Medicine, Christchurch Hospital, Christchurch, New Zealand \\ ${ }^{3}$ Department of Mathematics and Statistics, University of Canterbury, Christchurch, New Zealand
}

\begin{abstract}
Hyperglycaemia in critically ill patients increases the risk of further complications and mortality. A long-term verification of a model that captures the essential glucose- and insulin-kinetics is presented, using retrospective data gathered in an Intensive Care Unit (ICU). The model uses only two patient specific parameters, for glucose clearance and insulin sensitivity. The optimization of these parameters is accomplished through a novel integration-based fitting approach, and a piecewise linearization of the parameters. This approach reduces the non-linear, non-convex optimization problem to a simple linear equation system. The method was tested on long-term blood glucose recordings from 17 ICUpatients, resulting in an average error of $7 \%$, which is in the range of the sensor error. One-hour predictions of blood glucose data proved acceptable with an error range between 7 $11 \%$. These results verify the model's ability to capture longterm observed glucose-insulin dynamics in hyperglycaemic ICU patients.
\end{abstract}

Keywords-Critical Care, Glucose, Hyperglycemia, Insulin, Modeling.

\section{INTRODUCTION}

Critically ill patients often experience stress-induced hyperglycemia and high levels of insulin resistance, even if they have no history of diabetes [1-3]. Hyperglycemia can lead to an increased risk of further complications such as severe infections, myocardial infarctions, polyneuropathy and multiple organ failure [1, 4, 5]. Tight glucose control has been shown to reduce intensive care unit (ICU) patient mortality by as much as $45 \%$ [1]. To automate blood glucose management, models of insulin- and glucosekinetics are required that are capable of capturing long-term dynamics in a physiological realistic way. A new, physiologically verified model [6] and novel integrationbased fitting method are presented to evaluate the validity of the model over long periods using retrospective clinical data.

\section{Methodology}

\section{A. Model}

The basis for the presented model is the 3-compartment Minimal Model proposed by Bergman [7]. It was reduced to 2 compartments [8] and enhanced by additional dynamics [6, 9]. These additional dynamics account for an accumulation dynamic of insulin [10, 11], non-linear saturation of exogenous insulin appearance [12], and saturation of insulin utilisation and glucose clearance [13, 14]. To reduce model complexity and better match known physiological response, a term was included to suppress endogenous insulin secretion during periods of high exogenous insulin infusion [15]. The resulting model is defined:

$$
\begin{aligned}
& \dot{G}=-p_{G} G-S_{I}\left(G+G_{E}\right) \frac{Q}{1+\alpha_{G} Q}+P(t) \\
& Q=k \int_{0}^{t} I(\tau) e^{-k(t-\tau)} d \tau \\
& \dot{I}=-\frac{n I}{1+\alpha_{I} I}+\frac{u(t)}{V_{I}}+\frac{e^{-u(t)} I_{B}}{V_{I}}
\end{aligned}
$$

where $G=$ concentration of the plasma glucose above the basal level $(\mathrm{mmol} / \mathrm{L}), G_{E}=$ equilibrium level for plasma glucose concentration $(\mathrm{mmol} / \mathrm{L}), I=$ concentration of the plasma insulin above basal level (mU/L), $I_{B}=$ basal level for plasma insulin concentration $(\mathrm{mU} / \mathrm{L}), P(t)=$ exogenous glucose infusion rate $(\mathrm{mmol} /(\mathrm{L} \cdot \mathrm{min})), u(t)=$ insulin infusion rate $(\mathrm{mU} / \mathrm{min}), V_{I}=$ assumed insulin distribution volume (L), $n=$ delay in interstitial transfer of insulin $\left(\min ^{-1}\right), p_{G}=$ fractional clearance of plasma glucose at basal insulin $\left(\min ^{-1}\right)$ - time varying, $S_{I}=$ insulin sensitivity $(\mathrm{L} / \mathrm{mU} \cdot \mathrm{min})$ - time varying, $k=$ parameter controlling the effective half life of insulin $\left(\mathrm{min}^{-1}\right), \alpha_{I}=$ Michaelis-Menten parameter for insulin saturation, $\alpha_{G}=$ Michaelis-Menten parameter for glucose clearance saturation $(\mathrm{L} / \mathrm{mU})$.

This model has been physiologically verified over short 5 hour clinical trials for adaptive, targeted glucose control $[6,9]$.

\section{B. Parameter Fitting and Identification}

The objectives for the fitting method are low computation time, high accuracy for tracking changes in time varying patient specific parameters $\left(p_{G}, S_{I}\right)$ and physiologically realistic values of optimised parameters. A method that is convex and not dependent on starting points, as with non-linear recursive least squares (NRLS) [16], is also desirable.

Generic parameters $\left(V_{I}=12 \mathrm{~L}, n=0.16 \mathrm{~min}^{-1}, k=\right.$ $\left.0.0099 \mathrm{~min}^{-1}, \alpha_{G}=0.04 \mathrm{~L} / \mathrm{mU}, \alpha_{I}=0.0017 \mathrm{~L} / \mathrm{mU}\right)$ were found through extensive literature research [6] and are assumed to be constant. The exogenous feed details, $P(t)$, are known for each patient. The equilibrium glucose level, 
$G_{E}$, can be estimated by averaging the glucose readings across the prior 12 hours.

To identify $p_{G}$ and $S_{I}$, the $L_{2}$ norm between the measured data and the model output is minimized. Integration of (3) and substitution of the total measured glucose level $G_{T}=\left(G+G_{E}\right)$ and insulin saturation term $\bar{Q}=Q /\left(1+\alpha_{G} Q\right)$ delivers the following expression:

$$
\begin{aligned}
G_{T}(t) & -G_{T}\left(t_{0}\right)= \\
& =-\int_{t_{0}}^{t} p_{G}\left(G_{T}-G_{E}\right) d t-\int_{t_{0}}^{t} S_{I} G_{T} \bar{Q} d t+\int_{t_{0}}^{t} P d t
\end{aligned}
$$

To reduce computational complexity and account for a variation over time, the total time interval is divided into equal segments during which $p_{G}$ and $S_{I}$ are held constant, and are defined using heavyside step functions, $H(t)$.

$$
\begin{gathered}
p_{G}=\sum_{i=1}^{N} p_{G i}\left(H\left(t-t_{(i-1)}\right)-H\left(t-t_{i}\right)\right) \\
S_{I}=\sum_{i=1}^{N} S_{I i}\left(H\left(t-t_{(i-1)}\right)-H\left(t-t_{i}\right)\right)
\end{gathered}
$$

The only unknown parameters in (4), $p_{G i}$ and $S_{I i}$, are now constant over these time intervals and can be written in a simple linear equation system. The number of equations for each time segment can be arbitrarily selected:

$$
\underline{A}\left\{\begin{array}{c}
p_{G i} \\
S_{I i}
\end{array}\right\}=\underline{b}
$$

To make sure both $p_{G}$ and $S_{I}$ are within physiologically valid ranges, weighted constraints are placed on both parameters when solving (7).

To best compute the integrals in (4), the profile of $G_{T}$ is approximated using simple linear interpolation between the data points, forming a piecewise linear curve $G_{T-a p p r o x}$. The error between the patient's real glucose level $G_{T-\text { real }}$ and the approximated curve $G_{T-a p p r o x}$ is $\varepsilon$ :

$$
G_{T-\text { real }}(t)=G_{T-\text { approx }}(t)+\mathcal{E}(t) ; 0 \leq|\varepsilon(t)| \leq \delta ; \delta \in \text { small }
$$

Using (4), $G_{T \text {-real }}$ can be calculated in any given time interval $\left[\mathrm{t}_{0}, \mathrm{t}_{1}\right]$ with the constant parameters $\bar{p}_{G}$ and $\bar{S}_{I}$ :

$$
\begin{gathered}
G_{T-\text { real }}(t)=G_{T-\text { real }}\left(t_{0}\right)-\bar{p}_{G} \int_{t_{0}}^{t}\left(G_{T-\text { real }}(t)-G_{E}\right) d t \\
\quad-\bar{S}_{I} \int_{t_{0}}^{t} G_{T-\text { real }}(t) \bar{Q}(t) d t+\int_{t_{0}}^{t} P(t) d t \\
=G_{T-\text { approx }}\left(t_{0}\right)+\bar{p}_{G}\left(t-t_{0}\right) G_{E}-\bar{p}_{G} \int_{t_{0}}^{t} G_{T-\text { approx }}(t) d t \\
\quad-\bar{S}_{I} \int_{t_{0}}^{t} G_{T-\text { approx }}(t) \bar{Q}(t) d t+\int_{t_{0}}^{t} P(t) d t+E(t)
\end{gathered}
$$

Where the error term $E(t)$ is defined:

$$
\begin{aligned}
|E(t)| & =\left|\varepsilon\left(t_{0}\right)-\bar{p}_{G} \int_{t_{0}}^{t} \varepsilon(t) d t-\bar{S}_{I} \int_{t_{0}}^{t} \varepsilon(t) \bar{Q}(t) d t\right| \\
& \leq\left|\varepsilon\left(t_{0}\right)\right|+\bar{p}_{G}\left|\int_{t_{0}}^{t} \varepsilon(t) d t\right|+\bar{S}_{I}\left|\int_{t_{0}}^{t} \varepsilon(t) \bar{Q}(t) d t\right| \\
& \leq \delta+\bar{p}_{G} \int_{t_{0}}^{t}|\varepsilon(t)| d t+\bar{S}_{I} \int_{t_{0}}^{t}|\varepsilon(t)| \bar{Q}(t) d t \\
& \leq \delta+\bar{p}_{G} \delta\left(t-t_{0}\right)+\bar{S}_{I} \delta \int_{t_{0}}^{t} \bar{Q}(t) d t \\
& =O(\delta)
\end{aligned}
$$

Comparing the patient specific error terms to the corresponding integral terms from (9) shows that the error is small and the approximated curve does not compromise the quality of the optimisation utilising integral functions.

$$
\begin{aligned}
& \left|\frac{\bar{p}_{G} \delta\left(t-t_{0}\right)}{\bar{p}_{G} \int_{t_{0}}^{t} G_{T-\text { real }}(t) d t}\right|<\frac{\delta\left(t-t_{0}\right)}{\int_{t_{0}}^{t} 1 d t}=\delta \\
& \left|\frac{\bar{S}_{I} \delta \int_{t_{0}}^{t} \bar{Q}(t) d t}{\bar{S}_{I} \int_{t_{0}}^{t} G_{T-\text { real }}(t) \bar{Q}(t) d t}\right|<\frac{\delta \int_{t_{0}}^{t} \bar{Q}(t) d t}{\int_{t_{0}}^{t} \bar{Q}(t) d t}=\delta
\end{aligned}
$$

Thus, for a general time period $\left[\mathrm{t}_{0}, \mathrm{t}\right]$ the approximation $G_{T \text {-approx }}$ can be used to represent the real data between measurements with no significant error as long as blood glucose levels are $G_{T-\text { real }}(t)>1$, which is always true in hyperglycemia.

Integral functions also have the advantage of being insensitive to noise in the measured glucose data, effectively providing a low-pass filter in the summations involved in numerical integration.

\section{RESULTS}

For the long term data fitting and model verification, a random selection of 17 patients from a 201 patient data audit [6] with a period greater than one day and intervals between measured data points of three hours or less, were used. The data density of three hours was selected to ensure enough measurements to enable a good model evaluation. The entire length of stay was not always considered, as many patients only had a shorter period of data that fitted the criteria. This subset broadly represents the cross section of patients seen in the ICU, regarding medical condition, age, sex, APACHE II scores and mortality.

Two of these fits are shown in Fig. 1 and Fig. 2. To further reduce the noise in the data and the possibility of erroneous measurements, the fitted values of $p_{G}$ and $S_{I}$ were replaced by their smoothed 3-point moving average to produce the model curve in each figure. 
Patient 1090 had the lowest fitting error and standard deviation of the 17 patients considered, representing the best overall fit. Over the 1.6 days of the patient's 7-day stay, the insulin infused was constant at $1 \mathrm{U} / \mathrm{hr}$, and the feed was constant at $0.0247 \mathrm{~mol} /(\mathrm{L} \cdot \mathrm{min})$. Figure 1 shows a relatively constant normoglycemic profile over this time with low variations in insulin sensitivity, $S_{I}$, showing that the patient was very stable. The parameter $p_{G}$ was effectively constant at $0.02 \mathrm{~min}^{-1}$ where $S_{I}$ still shows some diurnal variation.

Patient 87 had a length of stay of 35 days, and over six consecutive days had sufficient data measurements for model fitting. Unlike patient 1090, the glucose level is much more variable, as is the insulin sensitivity, $S_{I}$. The glucose clearance, $p_{G}$, is stable over the time considered at $0.01 \mathrm{~min}^{-1}$, but does reach values of up to $0.015 \mathrm{~min}^{-1}$ on different days. These values are well within the physiological range expressed in the literature [13, 17-19],
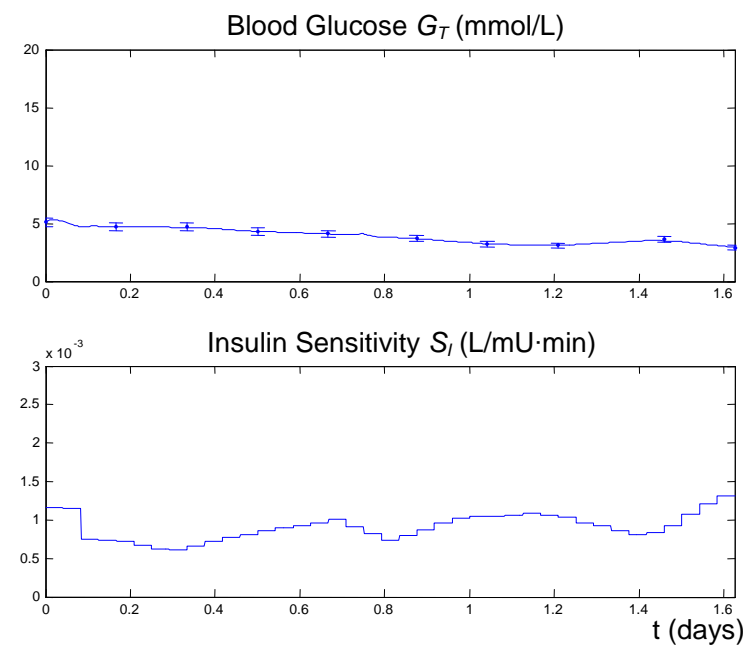

Fig. 1. Patient 1090 blood glucose data fit (top) and corresponding insulin sensitivity parameter $S_{I}$ (bottom).
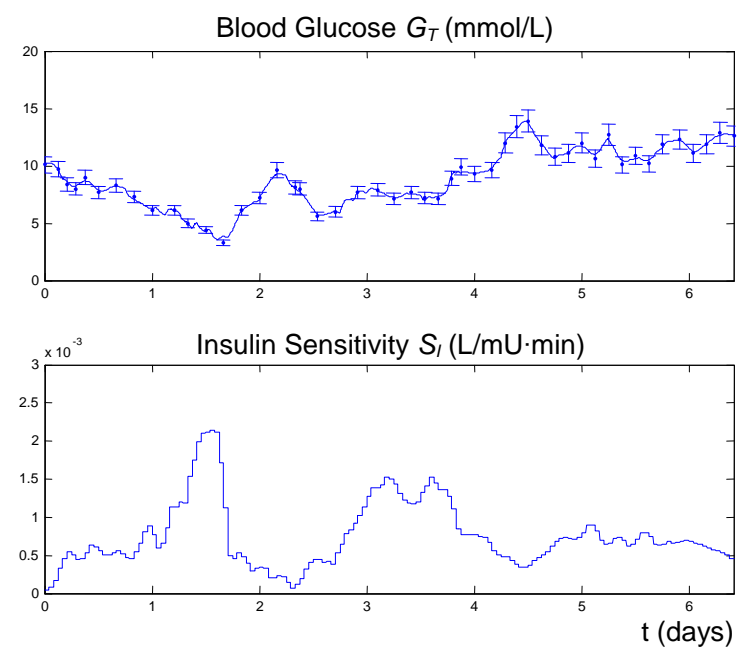

Fig. 1. Patient 87 blood glucose data fit (top) and corresponding insulin sensitivity parameter $S_{I}$ (bottom). and show a change in the metabolic responses at these time periods. Over the six days, the insulin infusion and glucose feed varied significantly as well.

To measure the error in data fitting, the "chi-square" quantity is calculated for the boundaries of the range of glucose measurement error (3.5-7\%) [20]. This reflects an error in the data fitting similar to the measurement error used in NRLS fitting [21]. The expected value of $\chi^{2}$ is $v=N$ $M$ where $N$ is the number of measurements and $M=2$ the number of parameters. The results are given in Table $\mathrm{I}$.

The average value of $v$ lies between $\chi_{3.5}^{2}$ and $\chi_{7}^{2}$, corresponding to a measurement error of $4.79 \%$, which is in the range of the reported sensor measurement error. The average verification interval was 3.1 days, with the longest at 12.3 days. The average fitting error over all patients was $4.39 \%$, with a range of $1.03-7.62 \%$. These values are also within the experimental error.

To test the prediction capability of the model, data from three patients who had a number of measurements one hour apart, was used. The fit from the previous eight hours was used and the parameters $p_{G}, S_{I}$ and $G_{E}$ were held constant over the next hour. The predicted value was then compared to the actual data, giving the percentage error $e$. This process was then repeated in moving 9 hour blocks for 15-25 Predictions per patient. The results in Table II show that one-hour predictions are on average $7-11 \%$ in error, which is also close to the measurement error.

TABLE I

ERRORS IN DATA FITTING

\begin{tabular}{|c|c|c|c|c|}
\hline $\begin{array}{r}\text { Patient } \\
\text { Number }\end{array}$ & $\begin{array}{r}\text { Time } \\
\text { interval } \\
\text { (days) }\end{array}$ & $\chi_{3.5}^{2}$ & $\chi_{7}^{2}$ & $v$ \\
\hline 24 & 2.0 & 57.82 & 14.46 & 29 \\
\hline 87 & 6.4 & 21.73 & 5.43 & 45 \\
\hline 130 & 1.4 & 142.20 & 35.55 & 28 \\
\hline 229 & 10.0 & 213.64 & 53.41 & 75 \\
\hline 289 & 1.7 & 49.52 & 12.38 & 10 \\
\hline 468 & 1.7 & 13.13 & 3.28 & 14 \\
\hline 484 & 1.7 & 8.88 & 2.22 & 15 \\
\hline 486 & 1.6 & 12.30 & 3.076 & 13 \\
\hline 519 & 12.3 & 62.73 & 15.68 & 79 \\
\hline 554 & 2.3 & 87.42 & 21.85 & 37 \\
\hline 666 & 1.6 & 15.48 & 3.87 & 12 \\
\hline 847 & 1.5 & 19.67 & 4.92 & 9 \\
\hline 1016 & 1.6 & 16.55 & 4.14 & 10 \\
\hline 1025 & 1.7 & 20.74 & 5.19 & 12 \\
\hline 1090 & 1.6 & 0.78 & 0.19 & 7 \\
\hline 1099 & 1.6 & 23.44 & 5.86 & 11 \\
\hline 1125 & 1.7 & 10.64 & 2.66 & 8 \\
\hline Average & 3.1 & 45.69 & 11.42 & 24.35 \\
\hline
\end{tabular}


TABLE II

MODEL PREDICTION ERRORS

\begin{tabular}{rrr}
\hline Patient & $\begin{array}{r}\text { Avg. prediction } \\
\text { error } \boldsymbol{e} \text { [\%] }\end{array}$ & $\begin{array}{r}\text { Error standard } \\
\text { deviation [\%] }\end{array}$ \\
\hline 24 & 6.77 & 4.68 \\
\hline 130 & 10.92 & 10.02 \\
\hline 554 & 10.73 & 9.79 \\
\hline
\end{tabular}

\section{DISCUSSION}

The fitting method proves to be very effective in reducing the non-linear, non-convex optimization problem to a simple linear equation system, which is not starting point dependent. By constraining the parameter optimization to physiologically valid ranges, the resulting values for $p_{G}$ and $S_{I}$ are valid. The glucose clearance $p_{G}$ remained nearly constant throughout all patients, while $S_{I}$ showed a greater variation, which matches reported physiological behavior [22]. The average fitting error is in the range of the measurement error of the sensor and matches the expected $\chi^{2}$ value. The low long-term-fit error values validate the models ability to capture the highly variable dynamics of hyperglycemic ICU patients over long periods.

The prediction of the glucose values for a period of one hour ahead were within $7-11 \%$ of the actual value, further validating the $p_{G}$ and $S_{I}$ values. Note that holding these parameters constant during the prediction interval is a conservative choice, as it ignores the prior changes in value.

\section{CONCLUSION}

The parameter identification method shows promise for similar drug therapy models and control algorithms. The optimized model was able to capture the dynamics in the measured data to within the $7 \%$ measurement error of the sensor, and was tested for time periods up to two weeks. The model provides a prediction for one hour with an error between $7-11 \%$, which is acceptable for control applications in a clinical setting. Due to the low computational complexity of the optimization algorithm, it is also well suited for real-time clinical control applications.

\section{REFERENCES}

[1] G. Van den Berghe, P. Wouters, F. Weekers, C. Verwaest, F. Bruyninckx, M. Schetz, et al., "Intensive insulin therapy in the critically ill patients," N Engl J Med, vol. 345, no. 19, pp. 13591367, 2001

[2] G. Van den Berghe, P. J. Wouters, R. Bouillon, F. Weekers, C. Verwaest, M. Schetz, et al., "Outcome benefit of intensive insulin therapy in the critically ill: Insulin dose versus glycemic control," Crit Care Med, vol. 31, no. 2, pp. 359-366, 2003.

[3] J. S. Krinsley, "Association between hyperglycemia and increased hospital mortality in a heterogeneous population of critically ill patients," Mayo Clin Proc, vol. 78, no. 12, pp. 1471$8,2003$.
[4] B. R. Bistrian, "Hyperglycemia and Infection: Which is the Chicken and Which is the Egg?," JPEN J Parenter Enteral Nutr, vol. 25, no. 4, pp. 180-181, 2001.

[5] S. E. Capes, D. Hunt, K. Malmberg, and H. C. Gerstein, "Stress hyperglycaemia and increased risk of death after myocardial infarction in patients with and without diabetes: a systematic overview," Lancet, vol. 355, no. 9206, pp. 773-778, 2000.

[6] C. V. Doran, "Modelling and Control of Hyperglycemia in Critical Care Patients," Masters Thesis, Department of Mechanical Engineering, University of Canterbury, Christchurch, New Zealand, 2004

[7] R. N. Bergman, D. T. Finegood, and M. Ader, "Assessment of insulin sensitivity in vivo," Endocr Rev, vol. 6, no. 1, pp. 45-86, 1985.

[8] C. V. Doran, J. G. Chase, G. M. Shaw, K. T. Moorhead, and N H. Hudson, "Automated Insulin Infusion Trials in the Intensive Care Unit," Diabetes Technology and Therapeutics, vol. 6, no. 2, pp. 155-166, 2004.

[9] J. G. Chase, G. M. Shaw, J. Lin, C. V. Doran, C. E. Hann, M. B Robertson, et al., "Adaptive Bolus-Based Set-Point Regulation of Hyperglycemia in Critical Care," in IEEE Engineering in Medicine and Biology. San Fransisco, USA, 2004.

[10] C. Cobelli, A. Caumo, and M. Omenetto, "Minimal model SG overestimation and SI underestimation: improved accuracy by a Bayesian two-compartment model," Am J Physiol, vol. 277, no. 3 Pt 1, pp. E481-488, 1999.

[11] K. Turnheim and W. K. Waldhausl, "Essentials of insulin pharmacokinetics," Wien Klin Wochenschr, vol. 100, no. 3, pp. 65-72, 1988.

[12] B. Thorsteinsson, "Kinetic models for insulin disappearance from plasma in man," Dan Med Bull, vol. 37, no. 2, pp. 143-153, 1990.

[13] R. L. Prigeon, M. E. Roder, D. Porte, Jr., and S. E. Kahn, "The effect of insulin dose on the measurement of insulin sensitivity by the minimal model technique. Evidence for saturable insulin transport in humans," J Clin Invest, vol. 97, no. 2, pp. 501-507, 1996.

[14] A. Natali, A. Gastaldelli, S. Camastra, A. M. Sironi, E. Toschi, A. Masoni, et al., "Dose-response characteristics of insulin action on glucose metabolism: a non-steady-state approach," Am J Physiol Endocrinol Metab, vol. 278, no. 5, pp. E794-801, 2000

[15] R. A. DeFronzo, J. D. Tobin, and R. Andres, "Glucose clamp technique: a method for quantifying insulin secretion and resistance," Am J Physiol, vol. 237, no. 3, pp. E214-223, 1979.

[16] R. Hovorka and P. Vicini, "Parameter Estimation," in Modelling Methodology for Physiology and Medicine, E. Carson and C. Cobelli, Eds. London: Academic Press, 2001, pp. 107-151.

[17] S. M. Furler, E. W. Kraegen, R. H. Smallwood, and D. J. Chisholm, "Blood glucose control by intermittent loop closure in the basal mode: computer simulation studies with a diabetic model," Diabetes Care, vol. 8, no. 6, pp. 553-561, 1985.

[18] A. Avogaro, J. D. Bristow, D. M. Bier, C. Cobelli, and G. Toffolo, "Stable-label intravenous glucose tolerance test minimal model," Diabetes, vol. 38, no. 8, pp. 1048-1055, 1989.

[19] F. Bettini, A. Caumo, and C. Cobelli, "Minimal Models in Meallike Protocols: Simulation Studies to Assess Precision and Physiological Plausibility of Parameter Estimates," presented at IEEE 17th Annual Conference, 1995.

[20] Arkray Inc. 2001, "GlucocardTM Test Strip 2 Data Sheet." Japan: Arkray Inc.

[21] E. R. Carson and C. Cobelli, Modelling methodology for physiology and medicine. San Diego: Academic Press, 2001.

[22] M. E. Wilinska, L. J. Chassin, H. C. Schaller, T. R. Pieber, and R. Hovorka, "Modelling Insulin Lispro Kinetics during Physiological Conditions in Subjects with Type 1 Diabetes Treated by CSII," presented at World Congress on Biomedical Engineering and Medical Physics, Sydney, Australia, 2003. 